\title{
Angular Spectrum Evaluation Tool analysis of the Crown of Light diamond cut
}

\author{
Jose Sasian $^{\mathrm{a}, *}$ and Reuven Paikin ${ }^{\mathrm{b}}$ \\ ${ }^{a}$ University of Arizona, Wyant College of Optical Sciences, Tucson, Arizona, United States \\ ${ }^{b}$ Duiker Investments 142 (Pty) Ltd. t/a Almod Diamonds Namibia, Prosperita, Windhoek, \\ Namibia
}

\begin{abstract}
We analyze the Crown of Light (COL) cut using an angular spectrum evaluation tool. Several light performing features of the COL are discussed. In particular, it is found that the angular spectrum of the COL tends to be concentrated and that this maximizes the brilliance and sparkle probability when the COL is aimed at localized light sources. It is contended that the COL represents a novel paradigm in diamond cuts. A distinctive feature of the COL cut is its dome shaped crown. (C) The Authors. Published by SPIE under a Creative Commons Attribution 4.0 Unported License. Distribution or reproduction of this work in whole or in part requires full attribution of the original publication, including its DOI. [DOI: 10.1117/1.OE.59.12.124104]
\end{abstract}

Keywords: light performance; diamond cut; gemstone brilliance; gemstone evaluation.

Paper 20200787 received Jul. 1, 2020; accepted for publication Nov. 25, 2020; published online Dec. 14, 2020.

\section{Introduction}

Human creativity is boundless, and when it is applied to diamond cuts, clever diamond cutters have proven their creativity in designing several outstanding round diamond cuts. An innovative cut that breaks the status quo paradigm in round brilliant cuts is the Crown of Light cut, or simply COL. Typically, round diamond cuts exhibit a crown with a flat table. For example, the traditional Tolkowsky cut shown in Fig. 1 has a flat table of $~ 55 \%$ of the diameter of the stone with 33 facets in the crown and 24 in the pavilion for a total of 57 cut facets (excluding the culet and the girdle). The Tolkowsky cut has evolved into a variety of cuts with excellent light performance, yet it adheres to a light performance determined in part by the substantial flat table.

The COL cut shown in Fig. 2 breaks with traditional diamond cutting to achieve a new paradigm in round brilliant light performance. This paper provides a study on the light performance of the COL cut using angular spectrum evaluation tool (ASET) technology. Because of the striking differences of the COL cut, mainly a domed shaped crown, a number of significant insights in light performance are gleaned from this study. This paper shows that the COL has established a new paradigm in round brilliant light performance. In addition, this paper furthers our research of light performance in gemstones.

\section{Review of ASET Technology}

A gemstone's ability to exhibit the effects of brilliance, fire, and scintillation depends on the angular directions of light that bring light into an observer's eyes. ${ }^{1}$ The angular directions are known technically as the angular spectrum of a gemstone. The ASET instrument was developed to provide information about the angular spectrum of a gemstone. ${ }^{1}$ Light performance in gemstones has been a subject of interest in the jewelry industry. ${ }^{2,3}$

As shown in Fig. 3, the gemstone located in the bottom of this figure redirects light to an observer's eye from three major angular direction ranges. The green angles range from 0 to $45 \mathrm{deg}$, the red angles from 45 to $75 \mathrm{deg}$, and the blue angles from $75 \mathrm{deg}$ to $90 \mathrm{deg}$. Although subdividing the angular hemisphere into more ranges improves our knowledge of

*Address all correspondence to Jose Sasian, Jose.sasian@optics.arizona.edu 


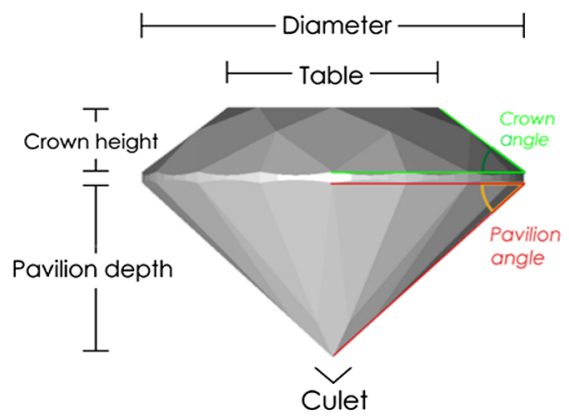

Fig. 1 Side view and nomenclature of the Tolkowsky cut with its characteristic flat table.
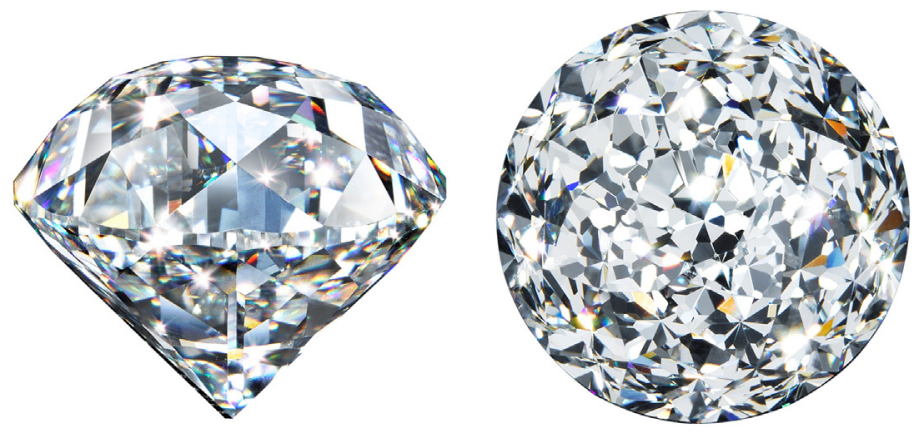

Fig. 2 The COL cut with its distinctive dome shaped crown.

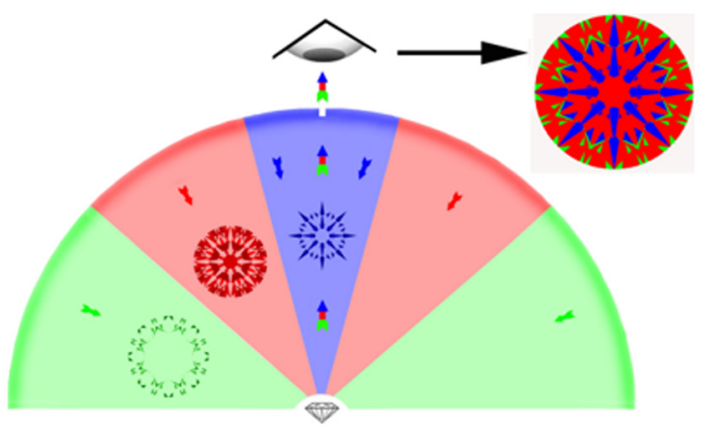

Fig. 3 Principles of ASET technology.

angular spectrum, the three ranges selected in the ASET instrument provide substantial information for evaluation purposes.

In Fig. 3, at the top right, the appearance of a round diamond under the ASET is shown. The blue region, in the face-up position of the stone, corresponds to the directions and light that could be blocked by the observer's head. It is desirable that the projection of the blue angular range is well distributed over the crown or top of the stone. This prevents large unilluminated areas and, most importantly, contributes to enhancing the facets that appear illuminated. This effect is known as contrast brilliance. ${ }^{4}$

The red angular range represents the directions that most likely makes the gemstone crown appear illuminated. This corresponds to light from lighting fixtures and windows high on the walls of a room. The green angular range represents the directions that are unlikely to make a stone appear brightly illuminated.

Under the ASET view, a gemstone that performs well has a large percentage (about 50\% to $70 \%$ ) of its area within the red range, a very well distributed and smaller percentage in blue (about $20 \%$ to $30 \%$ ), and a smaller percentage in green and in light leakage. 
The red areas in the crown of a stone under the ASET view most often appear illuminated. This illumination, or brilliance, tends to be diffused because the red represents a large angular range that captures light from large areas of the ceiling or walls in a room. When there are localized light sources, such as spot lighting or chandeliers, some facets will light up with bright sparkles as light is brought directly to the observer's eye from a direct pathway to the light source; however, these sparkles will not likely be in large numbers.

Although the blue range under the face-up position represents light obscuration and contrast brilliance, it becomes a most important angular range when the gemstone is tilted. The blue range represents the areas on the stone crown that appear illuminated most frequently with sparkle and scintillation. This is due to the inherent light retroreflection and symmetry of gemstones. In addition, the substantially smaller ceiling area that the blue range represents in a room makes the proportion of light fixture area to such a blue ceiling area much larger than for the red range.

As we naturally direct and align our hand to maximize the sparkle and scintillation seen in a gemstone, the blue angular range, being small, has a maximum probability of being aimed to a chandelier or group of localized light sources, and then a large number of facets would simultaneously be lit, producing a substantial illumination effect.

Thus the blue range represents areas and facets that appear most likely illuminated with direct light from localized sources. It is the blue area, under stone tilt, that exhibits the most sparkle and scintillation.

Figure 4 shows the ASET images of three stones. The left and center stones do not have a desirable light performance. The stone on the right corresponds to the Tolkowsky cut, which is considered an excellent cut for light performance.

\section{Crown of Light Diamond Cut}

U.S. Patent 6,761,044 describes a novel gemstone cut that, after refinement, became the COL cut. With reference to Fig. 5, the crown includes an octagonal table that is surrounded by eight triangular star facets. Eight table bezels are disposed between the star facets, and eight midbezels are disposed between the table bezels. The gemstone's pavilion has eight concentrically

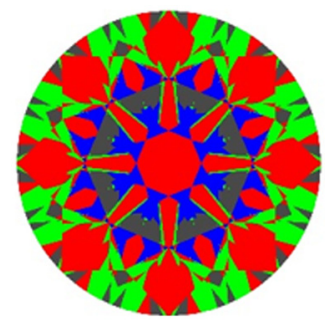

(a)

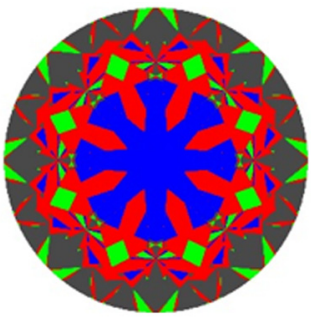

(b)

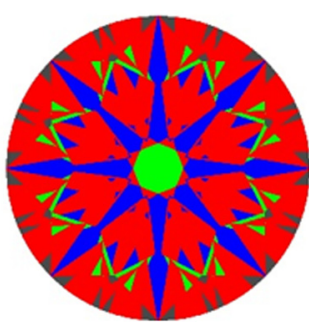

(c)

Fig. 4 ASET images of three round brilliant stones: (a) a stone with a significant green range, light leakage through the pavilion shown as grey areas and a small quantity of the blue; (b) a stone with major light leakage shown with gray color, a poor distribution of the blue range, and a very small percentage of the red range; and (c) a stone with a pleasing distribution of the blue range in an arrows pattern, a significant area of the red, and a small percentage of the green and light leakage.

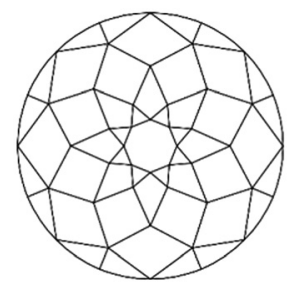

(a)

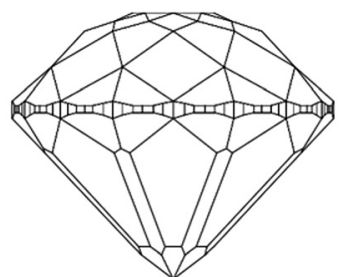

(b)

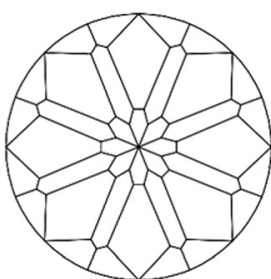

(c)

Fig. 5 Views of the cut facets in the COL cut. Note the dome shaped crown. 


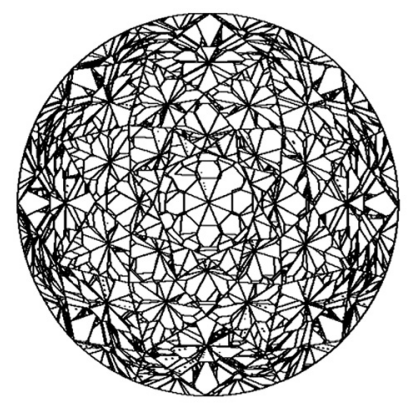

(a)

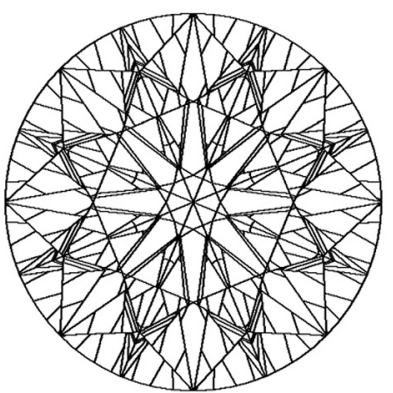

(b)

Fig. 6 Virtual facets for the (a) COL cut and (b) Tolkowsky cut for the face-up position.

Table 1 Virtual facet count

\begin{tabular}{|c|c|c|c|c|c|c|c|c|c|}
\hline \multicolumn{5}{|c|}{$\mathrm{COL}$} & \multicolumn{5}{|c|}{ Tolkowsky } \\
\hline Tilt (deg) & Blue & Red & Green & Gray & Tilt (deg) & Blue & Red & Green & Gray \\
\hline 0 & 6282 & 2906 & 519 & 1262 & 0 & 32 & 208 & 40 & 32 \\
\hline 5 & 5237 & 3766 & 550 & 1334 & 5 & 50 & 155 & 55 & 2 \\
\hline 10 & 2825 & 4976 & 736 & 1259 & 10 & 45 & 166 & 58 & 8 \\
\hline 15 & 1706 & 5286 & 1254 & 1258 & 15 & 31 & 174 & 48 & 18 \\
\hline 20 & 784 & 5076 & 1260 & 1745 & 20 & 35 & 173 & 58 & 21 \\
\hline 25 & 921 & 3777 & 1981 & 2202 & 25 & 28 & 175 & 54 & 27 \\
\hline
\end{tabular}

arranged culet pavilion facets, a girdle pavilion facet, and a bottom small break facet that are disposed between the culet pavilion facets. The gemstone's girdle has eight left top half facets and eight right top half facets (located between the girdle bezels of the crown) and eight left bottom half facets and eight right bottom half facets (disposed between the girdle pavilion facets and bottom small break facets). Thus there are 49 crown facets and 41 pavilion facets, including the culet, for a total of 90 cut facets. Clearly, a major distinctive feature of the COL is the dome shaped crown.

The Tolkowsky round brilliant cut has 57 facets (excluding the culet). In comparison, the COL cut has 90 facets. The larger number of facets provides more paths for the stone to exhibit brilliance, fire, and scintillation. The virtual facets, ${ }^{1}$ which are the optical projections of the actual facets, are comparatively small and numerous in the COL cut as shown in Fig. 6 and given in Table 1. The virtual facets result as each beam of light entering the stone is partitioned by the physical cut facets and as the beam refracts in, reflects on, and refracts out of the stone. In Table 1, the gray virtual facet count accounts for facets that are not illuminated directly by the hemisphere because light is leaking through the stone pavilion. The virtual facet count in Table 1 is for facets larger than $0.03 \mathrm{~mm}$ because smaller facets at a viewing distance of $250 \mathrm{~mm}$ would not be resolved by the average observer. The facet counts are for several angular tilts of the stone with respect to the face-up position, which is listed at $0 \mathrm{deg}$. The number of cut facets in the COL cut is not unusual as other flat table, round brilliant cuts can have 91,129, or more cut facets.

\section{ASET Characterization of the Crown of Light}

A COL, 1.2 carat clear diamond gemstone, 6- $\mathrm{mm}$ in diameter, was scanned with a measuring machine from Sarine $e^{5}$ to acquire the stone geometry, and then ASET images were computer generated as shown in Fig. 7. From left to right, ASET images correspond to the view of face up and then with the stone tilted $5 \mathrm{deg}, 10 \mathrm{deg}, 15 \mathrm{deg}, 20 \mathrm{deg}$, and $25 \mathrm{deg}$. For reference 

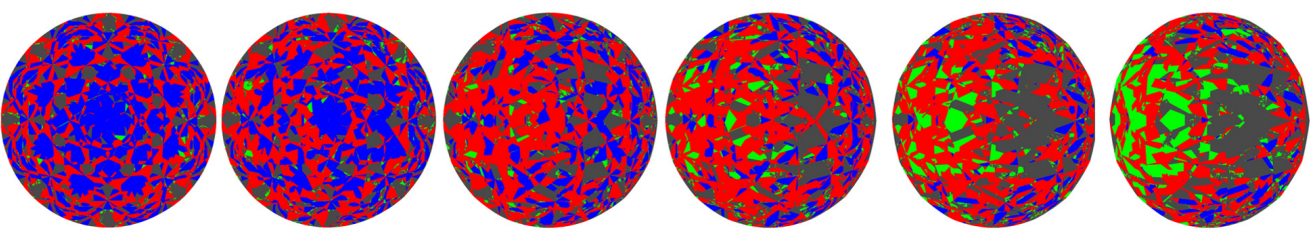

(a)
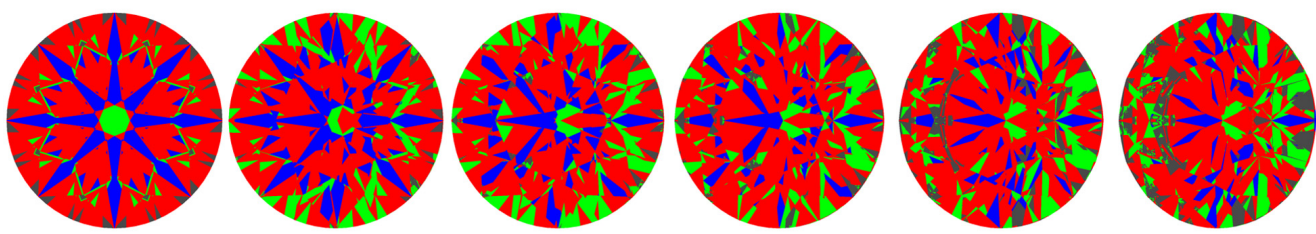

(b)

Fig. 7 ASET images of the (a) COL cut and (b) Tolkowsky cut. The leftmost image is the face-up view at $0 \mathrm{deg}$, and the subsequent images to the right are tilt views at $5 \mathrm{deg}, 10 \mathrm{deg}, 15 \mathrm{deg}$, 20 deg, and 25 deg.
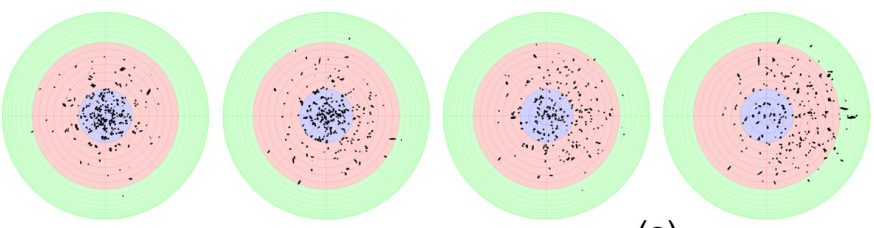

(a)
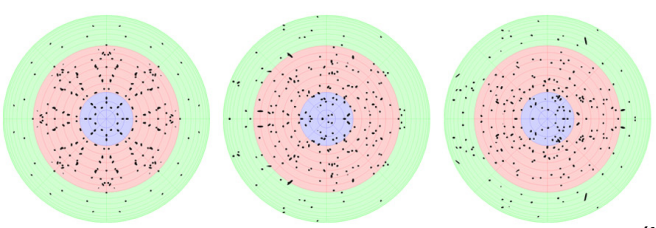

(b)
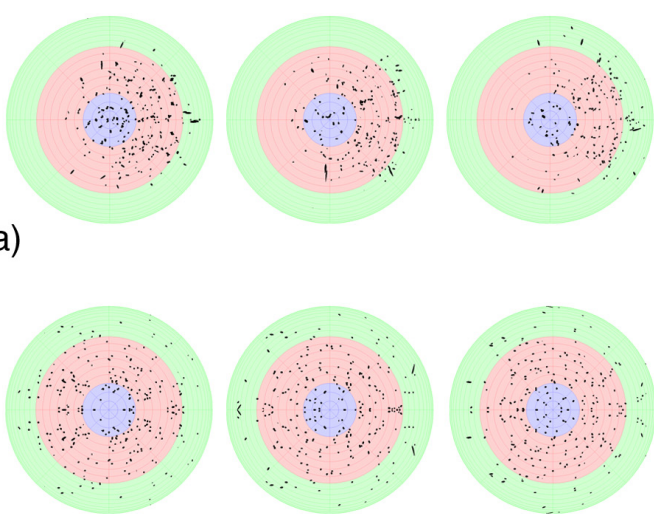

Fig. 8 Angular spectrum as projected on the hemisphere for the face-up position, $5 \mathrm{deg}, 10 \mathrm{deg}$, $15 \mathrm{deg}, 20 \mathrm{deg}$, and 25 deg. (a) COL cut images and (b) Tolkowsky cut images.

purposes, images corresponding to the Tolkowsky cut are also shown. The ASET images for the COL stone were generated from data of a real stone. The ASET images for the Tolkowsky cut were generated from a perfect geometrical model. The angular spectrum represented by black dots as projected in the illuminating hemisphere is shown in Fig. 8 for the COL cut on top and the Tolkowsky cut on bottom.

Figures 7 and 8 show two different projections of the angular spectrum, one on the stone crown and the other on the illuminating hemisphere. Table 2 provides data of angular spectrum distribution by angular range color for both cuts and for $0 \mathrm{deg}, 5 \mathrm{deg}, 10 \mathrm{deg}, 15 \mathrm{deg}, 20 \mathrm{deg}$, and $25 \mathrm{deg}$ stone tilts. The gray color represents light leakage through the stone pavilion. Table 3 provides data about the centroid and normalized spread of the angular spectrum in the hemisphere for both cuts. The normalized spread is a measure of the area occupied by the angular spectrum in the hemisphere. For the COL, the centroid shifts as the stone is tilted and the spread slightly increases. For the Tolkowsky cut, the centroid remains substantially centered, and the spread also remains the same as the stone is tilted.

A measure of a stone's capacity to exhibit fire is light dispersion. The images in Fig. 9 provide dispersion across the stone crown for the COL cut on top and for the Tolkowsky cut on bottom. Light dispersion is color-coded as dark orange, orange, light orange, and yellow, representing from least to most dispersion, respectively. The gray color indicates light leakage. The images in Fig. 9 were generated by tracing rays from the observer to the stone and determining light dispersion at two wavelengths. 
Table 2 Angular spectrum distribution

\begin{tabular}{|c|c|c|c|c|c|c|c|c|c|}
\hline \multicolumn{5}{|c|}{$\mathrm{COL}$} & \multicolumn{5}{|c|}{ Tolkowsky } \\
\hline $\begin{array}{l}\text { Tilt } \\
\text { (deg) }\end{array}$ & $\begin{array}{c}\text { Blue } \\
(\%)\end{array}$ & $\begin{array}{l}\text { Red } \\
(\%)\end{array}$ & $\begin{array}{c}\text { Green } \\
(\%)\end{array}$ & $\begin{array}{c}\text { Gray } \\
(\%)\end{array}$ & $\begin{array}{c}\text { Tilt } \\
\text { (deg) }\end{array}$ & $\begin{array}{c}\text { Blue } \\
(\%)\end{array}$ & $\begin{array}{l}\text { Red } \\
(\%)\end{array}$ & $\begin{array}{c}\text { Green } \\
(\%)\end{array}$ & $\begin{array}{c}\text { Gray } \\
(\%)\end{array}$ \\
\hline 0 & 45 & 34 & 2 & 19 & 0 & 21 & 68 & 6 & 5 \\
\hline 5 & 38 & 39 & 3 & 20 & 5 & 22 & 60 & 16 & 2 \\
\hline 10 & 24 & 48 & 3 & 25 & 10 & 18 & 61 & 19 & 2 \\
\hline 15 & 17 & 50 & 7 & 26 & 15 & 13 & 64 & 17 & 6 \\
\hline 20 & 10 & 46 & 12 & 32 & 20 & 11 & 61 & 17 & 11 \\
\hline 25 & 9 & 36 & 18 & 37 & 25 & 11 & 55 & 18 & 16 \\
\hline
\end{tabular}

Table 3 Angular spectrum centroid and normalized spread

\begin{tabular}{|c|c|c|c|c|c|}
\hline \multicolumn{3}{|c|}{$\mathrm{COL}$} & \multicolumn{3}{|c|}{ Tolkowsky } \\
\hline Tilt (deg) & Centroid (deg) & Spread & Tilt (deg) & Centroid (deg) & Spread \\
\hline 0 & 0 & 0.2 & 0 & 0 & 0.9 \\
\hline 5 & 5 & 0.3 & 5 & 4 & 0.8 \\
\hline 10 & 12 & 0.4 & 10 & 5 & 0.8 \\
\hline 15 & 20 & 0.5 & 15 & 4 & 0.8 \\
\hline 20 & 28 & 0.5 & 20 & 0 & 0.8 \\
\hline 25 & 30 & 0.5 & 25 & 0 & 0.8 \\
\hline
\end{tabular}
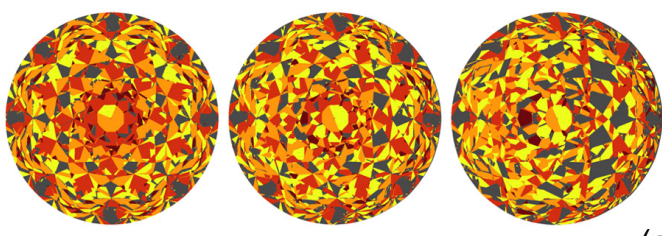

(a)
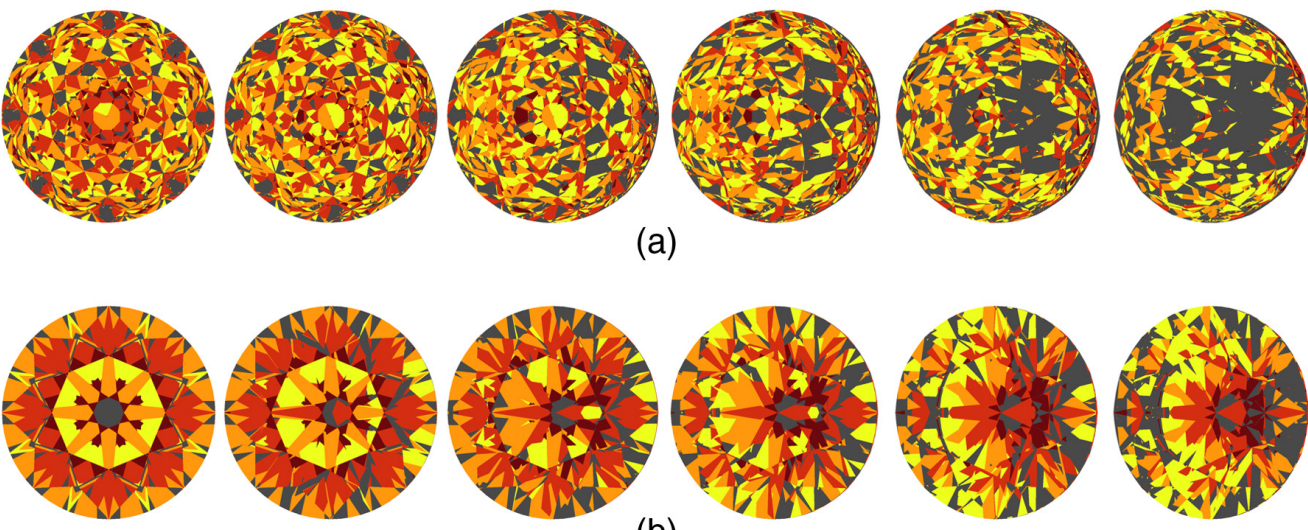

(b)
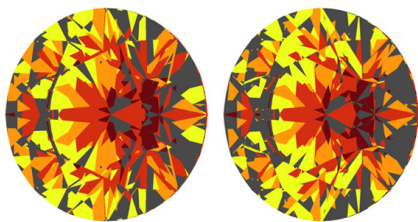

Fig. 9 Fire maps for the (a) COL and (b) Tolkowsky cut as projected in the stone crown for the face-up position, $5 \mathrm{deg}, 10 \mathrm{deg}, 15 \mathrm{deg}, 20 \mathrm{deg}$, and $25 \mathrm{deg}$ stone tilt.

Glare refers to the light that is directly reflected by the stone crown facets and that is not refracted inside the stone. Figure 10 shows glare maps across the crown for the face-up position for both the COL cut and the Tolkowsky cut. An observer who looks straight down at the COL cut would perceive light reflected by the crown facets coming from the blue, red, and green angular ranges in the illuminating hemisphere and for the Tolkowsky cut would perceive light from the blue and green angular ranges. The large flat table in the Tolkowsky cut can cause 


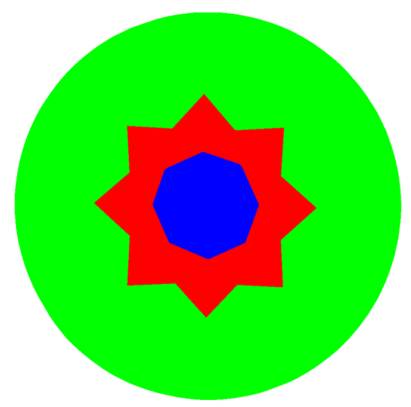

(a)

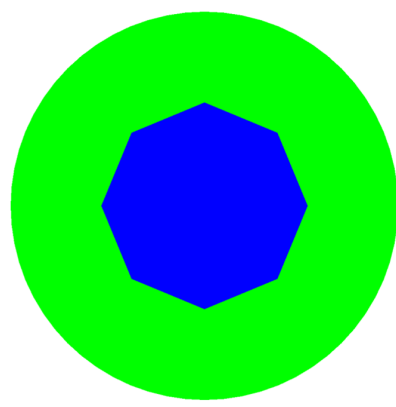

(b)

Fig. 10 Glare maps across the stone crown for the (a) COL and (b) Tolkowsky cut. The larger blue area in the Tolkowsky cut indicates a larger probability for the presence of glare due to sources in the blue angular range.

detrimental glare that interferes with observing light performance from light refraction in the stone.

\section{Discussion}

In examining a gemstone stone, the face-up position is important because it is a natural viewing position of an observer. Table 1 indicates that the COL cut creates more than one order of magnitude of virtual facets than the Tolkowsky cut. A larger number of virtual facets increases the light paths for light to create illumination effects in a gemstone. On the other hand, a smaller count of larger virtual facets can produce illumination effects over the larger facets that are appealing to an observer. With larger facets, the phenomenon of facet interplay can also take place, in which the appearance of the illuminated facets is reminiscent of views in a kaleidoscope. However, both types of cuts, with a small or large number of facets, are an option for gemstone cuts.

Table 2 provides the angular spectrum distribution for the $\mathrm{COL}$ and the Tolkowsky cuts. For the face-up position on the Tolkowsky cut, the distribution is $21 \%$ for the blue range, $68 \%$ for the red, $6 \%$ for the green, and $5 \%$ for light leakage, and on the COL cut, the distribution is $45 \%$ for the blue range, $34 \%$ for the red, $2 \%$ for the green, and $19 \%$ for leakage. These data indicate that the COL provides less diffuse brilliance because of its lower red color distribution, i.e., $68 \%$ versus $36 \%$, and suffers from a larger light leakage. However, the COL provides a larger blue color distribution of $45 \%$, which accounts for enhanced contrast brilliance.

The data in Table 2 indicate that, when the COL cut is tilted, light leakage increases, diffuse brilliance increases, and contrast decreases. This is shown clearly in the ASET images of Fig. 7. On the other hand, when the Tolkowsky cut is tilted, diffuse brilliance is maintained, contrast decreases, and light leakage increases, as also shown in Fig. 7.

The data in Table 2 also apply to the projection of the angular spectrum in the illuminating hemisphere of Fig. 8. For the COL cut, the angular spectrum tends to be concentrated, whereas in the Tolkowsky cut the angular spectrum is much more evenly distributed over the hemisphere. For example, the COL cut in the face-up position has a blue range of $45 \%$ versus $21 \%$ in the Tolkowsky cut. As the COL is tilted, the concentration moves from the hemisphere center to the hemisphere edge, tending to remain concentrated. As the Tolkowsky cut is tilted, its angular spectrum tends to remain evenly distributed over the hemisphere. This behavior is clearly shown in the hemisphere projection of Fig. 8 and in the data of Table 3 and is a major finding of this study.

The angular spectrum projection in the hemisphere clearly reveals the design strategy of a gem cutter to produce illumination effects. In the case of the COL, the gem cutter chose to maximize light performance relying on the observer's aiming of the stone.

Table 4 provides color-coded dispersion across the crown for the COL cut and for the Tolkowsky cut. These data are also for the face-up position and $5 \mathrm{deg}, 10 \mathrm{deg}, 15 \mathrm{deg}, 20 \mathrm{deg}$, and 25 deg stone tilts. 
Table 4 Light dispersion distribution

\begin{tabular}{|c|c|c|c|c|c|c|c|c|c|c|c|}
\hline \multicolumn{6}{|c|}{$\mathrm{COL}$} & \multicolumn{6}{|c|}{ Tolkowsky } \\
\hline $\begin{array}{l}\text { Tilt } \\
\text { (deg) }\end{array}$ & $\begin{array}{c}\text { Yellow } \\
(\%)\end{array}$ & $\begin{array}{c}\text { Yellow- } \\
\text { orange } \\
(\%)\end{array}$ & $\begin{array}{c}\text { Orange } \\
(\%)\end{array}$ & $\begin{array}{c}\text { Maroon } \\
(\%)\end{array}$ & $\begin{array}{c}\text { Gray } \\
(\%)\end{array}$ & $\begin{array}{c}\text { Tilt } \\
\text { (deg) }\end{array}$ & $\begin{array}{c}\text { Yellow } \\
(\%)\end{array}$ & $\begin{array}{c}\text { Yellow- } \\
\text { orange } \\
(\%)\end{array}$ & $\begin{array}{c}\text { Orange } \\
(\%)\end{array}$ & $\begin{array}{c}\text { Maroon } \\
(\%)\end{array}$ & $\begin{array}{c}\text { Gray } \\
(\%)\end{array}$ \\
\hline 0 & 18 & 28 & 28 & 5 & 21 & 0 & 12 & 36 & 31 & 9 & 12 \\
\hline 5 & 23 & 23 & 27 & 5 & 22 & 5 & 13 & 29 & 32 & 8 & 18 \\
\hline 10 & 23 & 23 & 22 & 4 & 28 & 10 & 10 & 30 & 30 & 8 & 22 \\
\hline 15 & 24 & 22 & 17 & 3 & 34 & 15 & 19 & 28 & 25 & 6 & 22 \\
\hline 20 & 22 & 21 & 11 & 2 & 44 & 20 & 21 & 20 & 25 & 6 & 28 \\
\hline 25 & 20 & 17 & 7 & 1 & 55 & 25 & 24 & 19 & 19 & 5 & 33 \\
\hline
\end{tabular}

The dispersion in the COL tends to be maintained as the stone tilts. This is also shown in Fig. 9, in which the stone appearance is similar as the stone tilts. The increased light leakage is also shown in Fig. 9. The phenomenon of facet interplay can be gleaned from the Tolkowsky cut dispersion maps in Fig. 9, in which the virtual facets appearance is reminiscent of the views in a kaleidoscope.

Thus the COL cut provides less diffuse brilliance, larger contrast, and more light leakage. However, it would be inappropriate to evaluate the COL cut in the same way as traditional cuts with a substantial flat table. Although traditional cuts have been optimized to perform over a wide range of illumination scenarios, the COL cut broke with tradition and was optimized to exhibit fire, scintillation, and sparkle at or near the face-up position when aimed at localized light sources. This is clearly shown by the images of the angular spectrum in Fig. 8, in which the angular spectrum for the COL cut near or at the face-up position tends to be concentrated at the blue angular range, while in the Tolkowsky cut, the angular spectrum is spread out throughout the illumination hemisphere. As schematically shown in Fig. 11, by having a concentrated angular spectrum, the COL cut has a larger probably of exhibiting scintillation and sparkle when it is aimed at localized light sources, which observers often do. This is because there are more directions within a smaller angular range that can coincide or be aligned with localized light sources, which light up the stone virtual facets.

It is clear that the angular spectrum representation of Fig. 8 calls for two distinct design choices of angular spectrum, namely spread out or concentrated. Alternatively, we can consider the angular spectrum to be projected on the stone crown, as shown in the ASET images of Fig. 7.

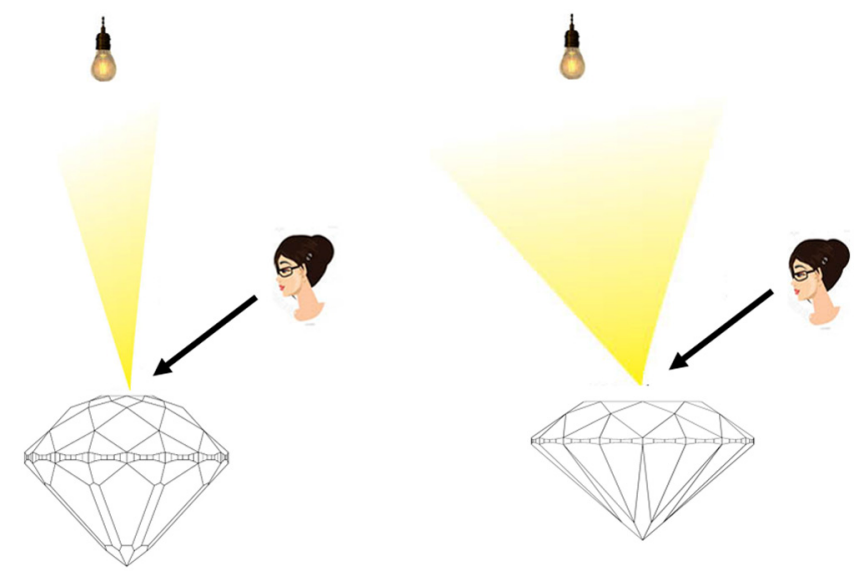

Fig. 11 Schematic representation of the angular spectrum of the COL and a standard Tolkowsky cut. The angular cone of rays is narrower for the COL than for the Tolkowsky cut, thereby increasing the probability of lighting virtual facets when the stone is aimed at localized light sources. 


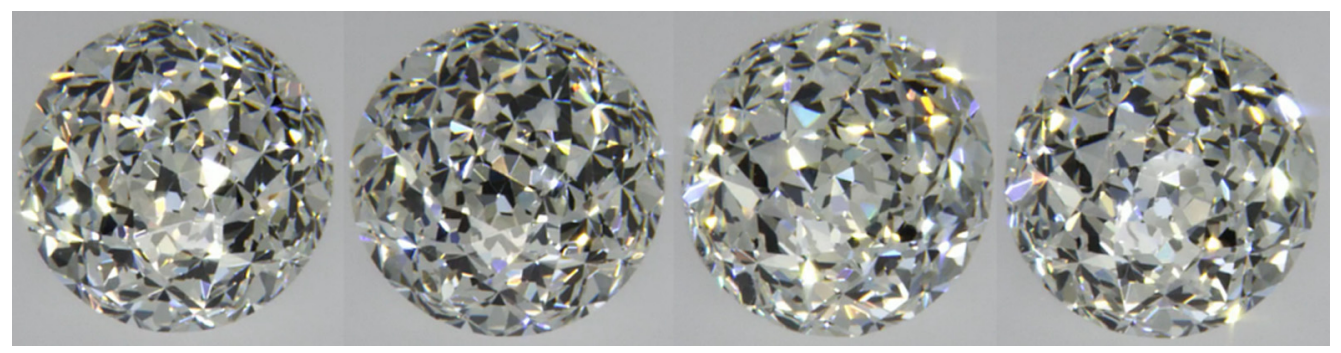

Fig. 12 Light performance of COL cut exhibiting substantial contrast brilliance, fire scintillation, and sparkle for several positions near the face-up position. The even distribution of these illumination effects over the stone crown is noteworthy.

The COL cut achieves a substantial mix of the blue and red angular ranges, which promotes the phenomenon of contrast brilliance. Further, the wide angular coverage by glare, and the fact that the COL cut has 49 crown cut facets, makes glare positively contribute to sparkle. As shown by the light dispersion maps of Fig. 9 and data of Table 4, dispersion is also well distributed over the crown of the COL cut. In sum, at or near the face-up position, the COL cut has an excellent distribution of contrast brilliance, glare, and dispersion. These features are shown in the photographs of a COL cut in Fig. 12. The result is a gemstone that is optimized for contrast brilliance, fire, scintillation, and sparkle at or near the face-up position and thus represents a new paradigm in gemstone light performance.

The light performance of the COL cut at high angular ranges is a trade-off with diffuse brilliance and virtual facet size. Diffuse brilliance is the illuminated appearance of the gemstone crown under diffuse illumination, and it relates to the red angular range. The COL has a small percentage of the red angular range and therefore does not provide the same diffuse brilliance as, for example, the Tolkowsky cut does. The relatively small cut facets in the COL result in small virtual facets and therefore in small stones, for example of $1 / 4$ of a carat, fire and scintillation tend to become pinpoint events. One novel difference, however, is that the COL minimizes the detrimental table glare of traditional cuts because glare due to small facet size contributes to sparkle. The dome shaped crown of the COL cut is a distinctive feature.

The analysis of the COL cut in this paper corresponds to an actual very well-cut stone. When a stone is not well cut, its light performance is significantly degraded, as illustrated in Fig. 4 and discussed in detail in Ref. 1. For comparison purposes, Table 5 provides the angular spectrum distribution for the actual COL cut and for a geometrically perfect virtual model of the COL cut. Figures 13 and 14 show the projection of the angular spectrum on the stone crown and on the hemisphere for both cuts. As can be noted, the actual cut does not differ significantly from the geometrical perfect virtual model. The projection of the angular spectrum in the hemisphere of the virtual model also supports our findings.

Table 5 Angular spectrum distribution

\begin{tabular}{|c|c|c|c|c|c|c|c|c|c|}
\hline \multicolumn{5}{|c|}{$\mathrm{COL}$} & \multicolumn{5}{|c|}{ Virtual COL } \\
\hline $\begin{array}{l}\text { Tilt } \\
\text { (deg) }\end{array}$ & $\begin{array}{c}\text { Blue } \\
(\%)\end{array}$ & $\begin{array}{l}\text { Red } \\
(\%)\end{array}$ & $\begin{array}{c}\text { Green } \\
(\%)\end{array}$ & $\begin{array}{c}\text { Gray } \\
(\%)\end{array}$ & $\begin{array}{c}\text { Tilt } \\
\text { (deg) }\end{array}$ & $\begin{array}{c}\text { Blue } \\
(\%)\end{array}$ & $\begin{array}{l}\text { Red } \\
(\%)\end{array}$ & $\begin{array}{c}\text { Green } \\
(\%)\end{array}$ & $\begin{array}{c}\text { Gray } \\
(\%)\end{array}$ \\
\hline 0 & 45 & 34 & 2 & 19 & 0 & 46 & 36 & 1 & 17 \\
\hline 5 & 38 & 39 & 3 & 20 & 5 & 37 & 41 & 2 & 20 \\
\hline 10 & 24 & 48 & 3 & 25 & 10 & 22 & 50 & 4 & 24 \\
\hline 15 & 17 & 50 & 7 & 26 & 15 & 16 & 50 & 8 & 26 \\
\hline 20 & 10 & 46 & 12 & 32 & 20 & 10 & 48 & 11 & 31 \\
\hline 25 & 9 & 36 & 18 & 37 & 25 & 9 & 37 & 18 & 36 \\
\hline
\end{tabular}



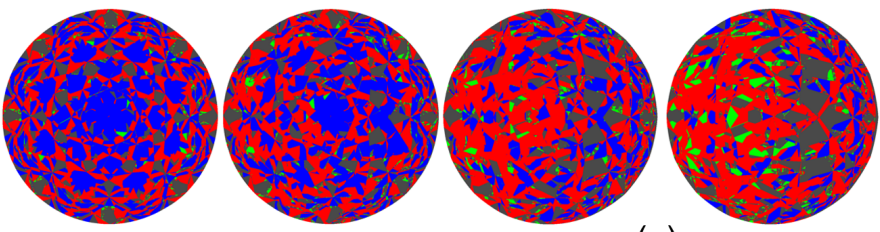

(a)
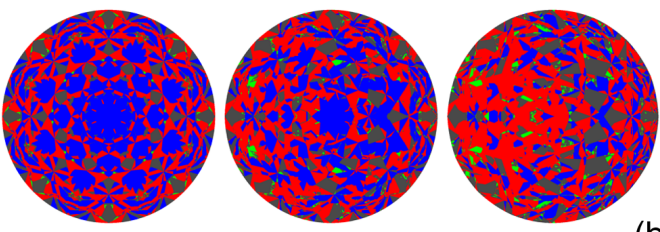

(b)

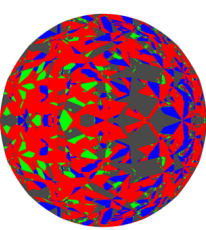

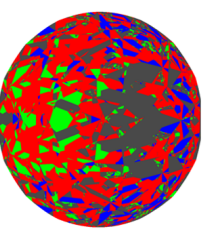
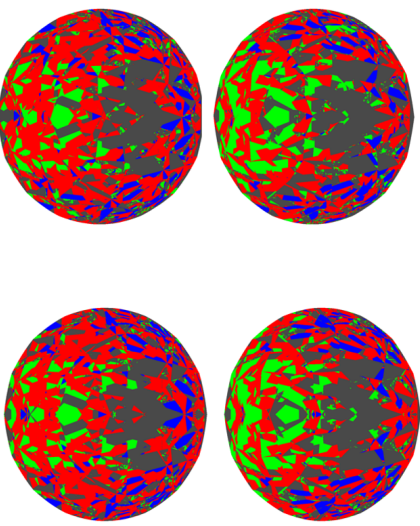

Fig. 13 ASET images of the (a) COL cut and (b) perfect geometry virtual model cut. The leftmost image is the face-up view at $0 \mathrm{deg}$, and the subsequent images to the right are tilt views at 5 deg, $10 \mathrm{deg}, 15 \mathrm{deg}, 20 \mathrm{deg}$, and $25 \mathrm{deg}$.
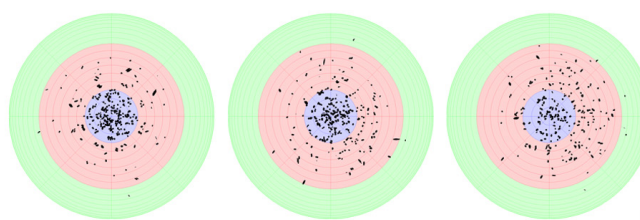

(a)
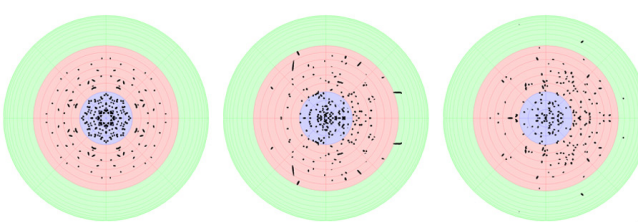

(b)
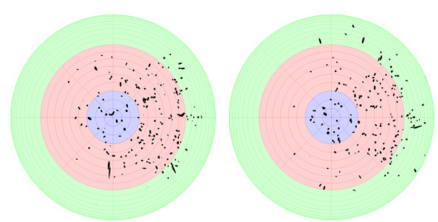

)
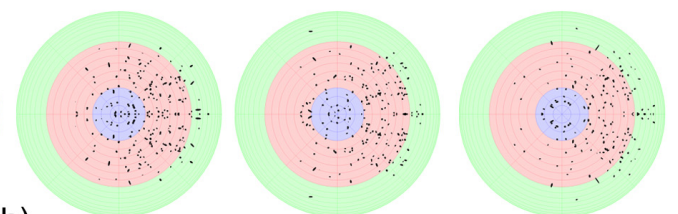

Fig. 14 Angular spectrum images projected on the hemisphere of (a) the COL cut and (b) perfect geometry virtual model cut. The leftmost image is the face-up view at 0 deg, and the subsequent images to the right are tilt views at $5 \mathrm{deg}, 10 \mathrm{deg}, 15 \mathrm{deg}, 20 \mathrm{deg}$, and $25 \mathrm{deg}$.

\section{Conclusion}

In this paper, we have used ASET technology to analyze the COL cut in comparison with the traditional Tolkowsky cut. A find is that technically the COL embodies a new paradigm in light performance with the cut being designed for maximum contrast brilliance, fire, scintillation, and sparkle at or near the face-up position. The angular spectrum as projected on the illuminating hemisphere tends to be localized rather that extended. This leads to substantially improved sparkle and scintillation performance when the cut is aimed at localized light sources. The increased number of cut facets results in an increased number of light paths for the cut to create illumination effects when the stone is aimed at localized light sources. Another find is the even distribution of illumination effects across the crown that under precarious spot lighting conditions, which are typical, the COL cut outperforms cuts with large flat tables in fire, scintillation, and sparkle. The crown of the COL is not truncated but features a distinctive dome shape. Overall, the COL cut brings up a novel light performance paradigm. The light performance benefits of the COL cut come, however, with a trade-off of reducing diffuse brilliance and virtual facet size. This paper furthers our research in light performance in gemstones and illustrates the use of ASET technology. ${ }^{1}$

\section{Acknowledgments}

The American Gems Society Labs kindly provided the ASET images and data presented in this paper. The authors declare no conflicts of interest. 


\section{References}

1. J. Sasian et al., "Evaluation of brilliance, fire, and scintillation in round brilliant gemstones," Opt. Eng. 46(9), 093604 (2007).

2. T. S. Hemphill et al., "Modeling the appearance of the round brilliant cut diamond: an analysis of brilliance," Gems Gemol. 34(3), 158-183 (1998).

3. I. M. Reinitz et al., "Modeling the appearance of the round brilliant cut diamond: an analysis of fire, and more about brilliance," Gems Gemol. 37(3), 174-197 (2001).

4. M. Cowing, "Diamond brilliance: theories, measurement and judgement," J. Gemmol. 27(4), 209-227 (2000)

5. Sarine Company, "Dimension HD," 2020, https://sarine.com/products/diamension-hd/

Jose Sasian is a professor at Wyant College of Optical Sciences of the University of Arizona. His research interests are optical engineering, education in optics, art in optics and optics in art, optical design, lithography, optomechanics, optical testing and fabrication, light in gemstones, and light propagation.

Reuven Paikin has over four decades of diamond industry expertise. He is the global director of Almod Diamonds Rough Manufacturing and oversees 350 employees across four Crown of Light cut/polish factories in Namibia, Ukraine, Canada, and New York. As the director of manufacturing and a Master Cutter, he plans and implements start-to-finish training programs for the Crown of Light premium diamond. His oversight includes quality evaluation, production control, quality, and final yield ratio. 